\title{
Influencia del corredor bucal y la exposición gingival en la percepción estética de la sonrisa
}

\author{
Influence of buccal corridor and gingival display in the perception esthetic of the smile \\ Katherine Rose Espinoza-Barco ${ }^{1, a}$, Katty Ríos-Villasis ${ }^{2, a, b}$, Carlos Liñán-Durán ${ }^{3, a, b, c}$
}

\section{RESUMEN}

Objetivo: Evaluar la influencia del corredor bucal y la exposición gingival en la percepción estética de la sonrisa de personas comunes pertenecientes a la Escuela de Sanidad Naval de la Marina de Guerra del Perú (ESNA - MGP), Bachilleres en Estomatología y Residentes de Ortodoncia de la Facultad de Estomatología de la Universidad Inca Garcilaso de la vega (FEST-UIGV). Materiales y Métodos: Se evaluó 105 personas con 35 sujetos por grupo evaluado. Se utilizó la fotografía de la sonrisa de una mujer, la cual fue modificada mediante el programa Adobe Photoshop con exposiciones gingivales (EG) de $0 \mathrm{~mm},+2 \mathrm{~mm},+4 \mathrm{~mm}$ y corredor bucal (CB): estrecho; amplio y nulo. La percepción de los grupos de evaluadores fue determinada mediante una escala visual análoga (EVA). Resultados: Se encontraron diferencias estadísticamente significativas entre los grupos (Anova-Tukey; $\mathrm{p}<0.05$ ). Personas comunes, Bachilleres y Residentes prefieren sonrisas con EG 0mm y CB estrechos a nulos. Considerando sonrisas con EG 4mm y CB amplios, como sonrisas no atractivas. Conclusión: Tener CB mínimos o estrechos y EG de 0mm; es una característica estética preferida por la mayoría de personas comunes, mientras que las EG de $4 \mathrm{~mm}$ y los CB amplios se deberían incluir en la lista de problemas durante el diagnóstico y el plan de tratamiento ortodóntico.

PALABRAS CLAVE: Percepción, estética dental, sonrisa. (DeCS, BIREME)

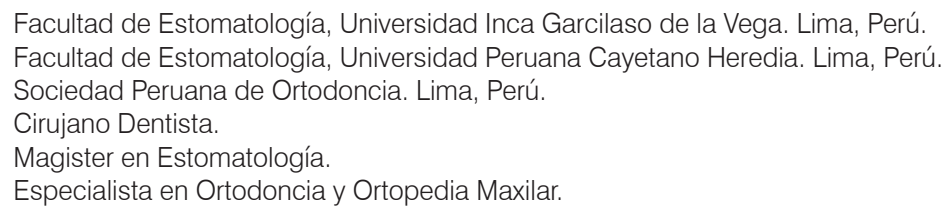




\section{SUMMARY}

Objective: To evaluate the influence of buccal corridor and smile gingival on smile esthetics perception of laypersons from the Naval School of Health of the Navy of Peru (ESNA-MGP), graduates and residents of orthodontic (FEST-UIGV). Methods: The sample consisted of 105 persons with 35 subjects by group evaluator. To carry out the study was used a photography of the face during the smile of a woman with parameters close to ideal, which was modified by a computer specialist by the Adobe Photoshop program on 3 different levels of gingival exposure (EG): $0 \mathrm{~mm},+2 \mathrm{~mm},+4 \mathrm{~mm}$ and 3 different types of buccal corridor (CB): narrow, wide and null. The perception of the three groups of raters was determined by a visual analog scale (VAS). Results: They found statistically significant differences among groups (Anova-Tukey; $p<0.05$ ). Laypersons, graduates and residents prefer smiles with EG Omm and CB narrows-null. Considering the smiles with EG $4 \mathrm{~mm}$ and $C B$ wides as unattractive smiles. Conclusion: $C B$ minimal or narrow and EG Omm; is a preferred aesthetic characteristic shared by most laypersons, while EG $4 \mathrm{~mm}$ and $C B$ wide should be included in the list of issues during diagnosis and orthodontic treatment plan.

\section{KEYWORDS: Perception, esthetics dental, smile. (MeSH, NLM)}

\section{INTRODUCCIÓN}

Una de las características de la estética facial que sin duda resalta a la vista es la sonrisa, desafortunadamente los dientes no siempre están en balance con las estructuras faciales adyacentes (1). La sonrisa es legítimamente considerada un medio valioso no verbal de comunicación social y un criterio del atractivo facial. El atractivo facial y el atractivo de sonrisa parecen firmemente conectados entre sí, la sonrisa juega un papel importante en la expresión facial y la apariencia (2).

Los pacientes aspiran a mejorar su estética con el objeto de verse y sentirse mejor, por lo que es necesario proporcionarles una sonrisa armoniosa, agradable y balanceada, de lo contrario se puede afectar la percepción que se tiene de la persona.

Fue en el siglo XVIII que Alexander Baumgarten utilizó el término "aesthetica" en un tratado en latín sobre la belleza de la poesía, y poco después el término fue aplicado ampliamente a las artes y la naturaleza (3).

La estética facial es la encargada de estudiar el grado de belleza de la cara. No hay una única estética facial ideal pues varía con el discurrir del tiempo; es decir, la estética facial está sometida a modas, de manera que no existe una noción exclusiva de belleza facial sino una serie de conceptos diversos, en los que el rostro es apreciado desde percepciones diferentes (4). Desgraciadamente, la valoración estética depende en gran medida del punto de vista de quien la realiza. En general la estética va asociada a la proporcionalidad y a la armonía, siendo considerado lo desproporcionado como antiestético; mientras que, lo proporcionado es aceptable, aunque no siempre bello (5).

La percepción de la estética varía de persona a persona y está influenciada por las experiencias personales y ambientes sociales (6).

La ortodoncia es la especialidad de la odontología encargada del estudio con detalle de las características del rostro humano; siendo su objetivo primordial mejorar la estética facial (7). Dustin y col. (8) mencionan que Roden el año 2004 afirmaba que la percepción de la estética facial de los ortodoncistas variaba de manera significativa con relación a la percepción de aquellas personas sin preparación. Motivo por el cual, el profesional debe adoptar una filosofía de tratamiento y usar una evaluación subjetiva cuando trata al paciente. Si el ortodoncista tiene una percepción de la estética que no es congruente con la percepción del paciente, el resultado podría no ser aceptable para él, así su función haya sido mejorada.

Las nociones comunes acerca de la estética facial generalmente se basan en opiniones subjetivas en lugar de datos científicos comprobados. Calificación y cuantificación de belleza no son fáciles. Sin embargo, a pesar de su complejidad, una estimación de la percepción de la belleza es un requisito en ortodoncia ya que el diagnóstico y la planificación del 
tratamiento deben basarse en la evidencia científica de los estudios relacionados con la medición de la belleza (9).

Las preocupaciones de los pacientes sobre su rostro, o más particularmente por su sonrisa son generalmente influenciadas por su entorno social más que por los dentistas o los ortodoncistas. Se ha observado que la cultura, la posición social y el nivel de educación son factores que afectan considerablemente la evaluación de la estética (9). Por lo tanto, el resultado clínico obtenido después del tratamiento de ortodoncia puede ser juzgado de manera diferente por los pacientes y ortodoncistas. Esta diferencia en la percepción estética de la sonrisa hace que sea complicado especificar los criterios de satisfacción para el tratamiento de ortodoncia $(10,11)$. La estética facial y dental se han hecho cada vez más importantes durante la última década, tanto para los pacientes como para los clínicos en el momento en que se realiza el diagnóstico y planificación de tratamiento. Recientemente, el campo de la ortodoncia ha experimentado un gran cambio con respecto a la estética, con un gran énfasis sobre los tejidos blandos que se encuentra alrededor de la boca $(12,13)$. Nuevos datos relacionados con la ciencia estética surgen. Tres aspectos de la estética de la sonrisa recientemente han captado gran atención: la cantidad de exposición gingival, el arco de la sonrisa y el tamaño de corredor bucal (14).

Se han evaluado muchas variables que afectan la estética y el atractivo de la sonrisa; sin embargo, la influencia de la interacción de diversas variables no son bien conocidas. Por otra parte, las personas comunes ajenas a la profesión y los odontólogos ya sean generales o especialistas, pueden ver la estética de la sonrisa de manera diferente. Allí radica la importancia de unificar criterios y sobre todo conocer lo que estas personas comunes, que podrían ser nuestros pacientes, consideran estético y agradable.

El propósito de esta investigación fue evaluar la influencia del corredor bucal y la exposición gingival en la percepción estética de la sonrisa de personas comunes, Bachilleres en Estomatología y Residentes de Ortodoncia, a fin de identificar y unificar criterios; ya que tanto personas comunes como profesionales pueden ver la estética de la sonrisa de manera diferente, lo cual nos permitirá cumplir con las expectativas estéticas de las personas que acudan a realizarse un tratamiento de ortodoncia, brindando un tratamiento más satisfactorio.

\section{MATERIAL Y MÉTODOS}

Este fue un estudio observacional, descriptivo, transversal y comparativo, con una muestra no probabilística y por conveniencia. La muestra estuvo conformada por 105 personas: 35 personas comunes pertenecientes a la Escuela de Sanidad Naval de la Marina de Guerra del Perú, 35 Bachilleres en Estomatología y 35 Residentes de Ortodoncia de la Facultad de Estomatología de la Universidad Inca Garcilaso de la Vega, durante los meses de Julio a Agosto del 2014, de ambos sexos, con edades comprendidas entre 18 y 41 años de edad, que cumplieron con los criterios de inclusión y exclusión.

Se utilizó la fotografía de una mujer, la cual previamente fue notificada con el propósito del estudio. Una vez que aceptó participar en el estudio voluntariamente, firmó un consentimiento informado. Se le tomó varias fotografía utilizando una cámara Nikon D3100 apoyada sobre un trípode, contra una pared de fondo blanco a una distancia de $30 \mathrm{~cm}$ y a $1 \mathrm{~m}$ de la cámara con el rostro mirando al frente durante la sonrisa y el plano de Frankfort paralelo al piso. Adicionalmente, se tomó fotografías intraorales con retractores de carrillo, para obtener elementos como encía; que no fueron visibles en la fotografía de sonrisa. La instrucción fue tener el cabello amarrado, no usar aretes, no tener maquillaje, no cerrar los ojos, humedecerse los labios y sonreír. Luego de que un juicio de expertos seleccionó la fotografía más adecuada; esta fue modificada por un experto en computación mediante el programa Adobe Photoshop, con la finalidad de obtener variaciones en el nivel de la exposición gingival $(0 \mathrm{~mm},+2 \mathrm{~mm}$, $+4 \mathrm{~mm}$ ) y conjugarlas con la amplitud del espacio del corredor bucal (amplio, nulo, estrecho). Obteniendo un número de 9 con diferentes niveles en la exposición gingival y corredor bucal (Figura 1). Las fotografías modificadas, fueron impresas en papel fotográfico y dispuestas de forma aleatoria en un rotafolio para su posterior análisis.

A cada participante se le explicó el propósito del estudio y luego que aceptaron participar voluntariamente en la investigación se les indicó 


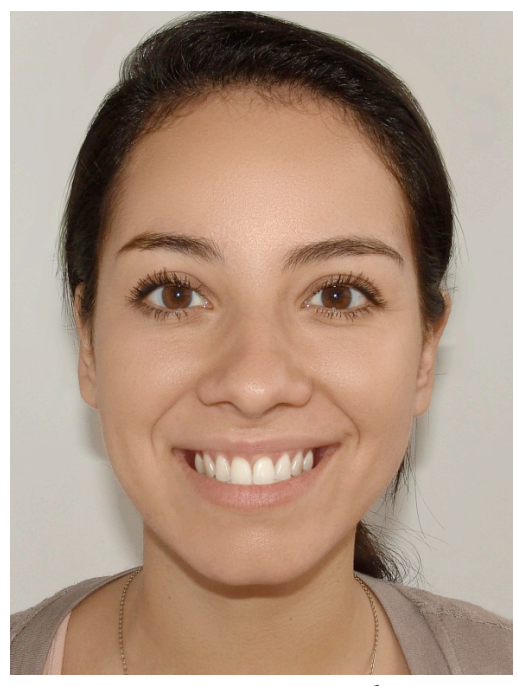

EG $2 \mathrm{~mm}-\mathrm{CB}$ amplio

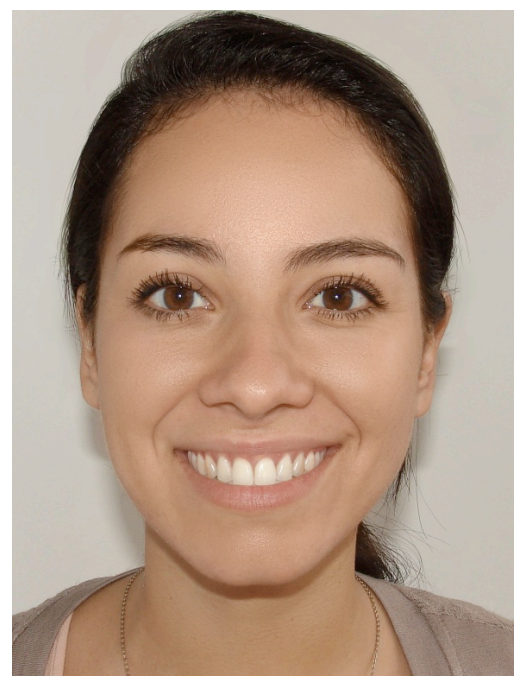

EG 2mm - CB nulo

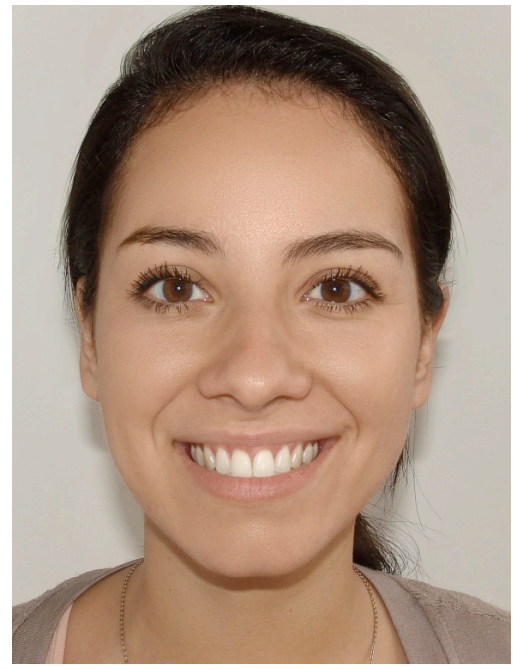

EG $2 \mathrm{~mm}-\mathrm{CB}$ estrecho

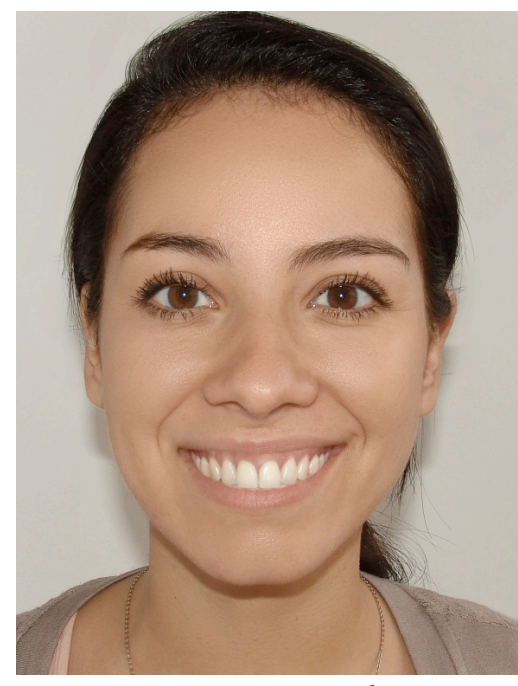

EG 4mm - CB nulo

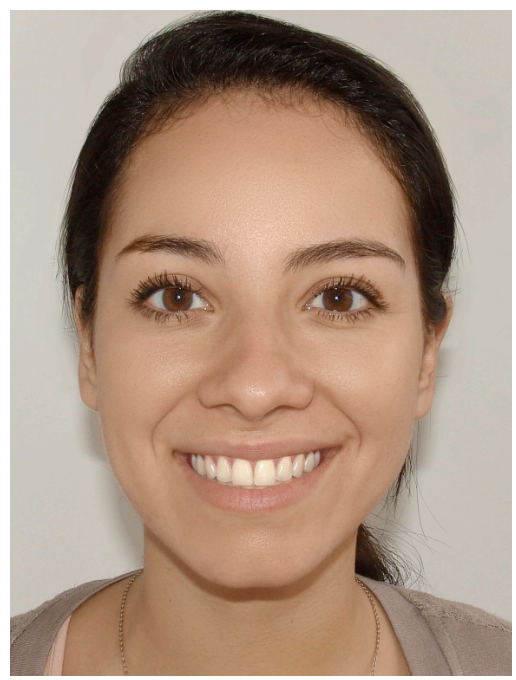

EG 0mm - CB estrecho

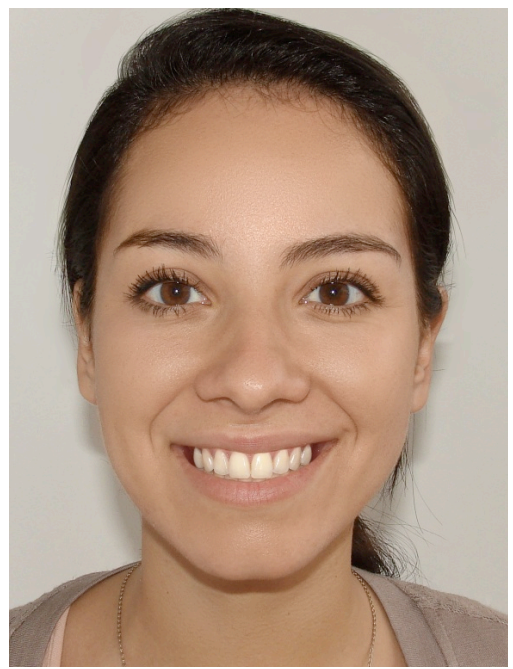

EG 0mm - CB amplio 


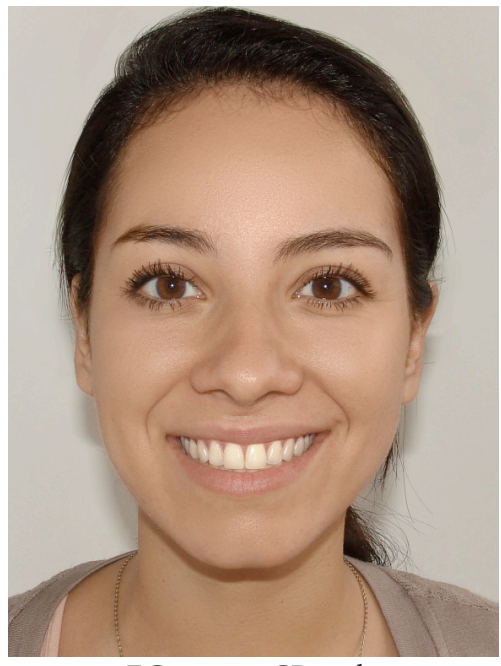

EG 0mm - CB nulo

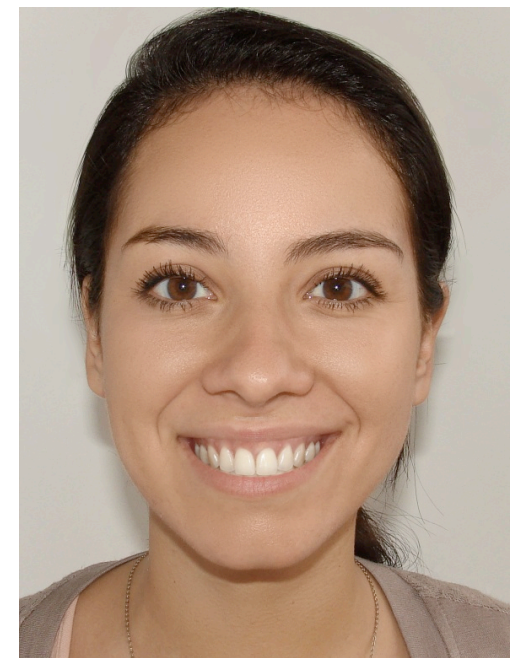

EG 4mm - CB estrecho

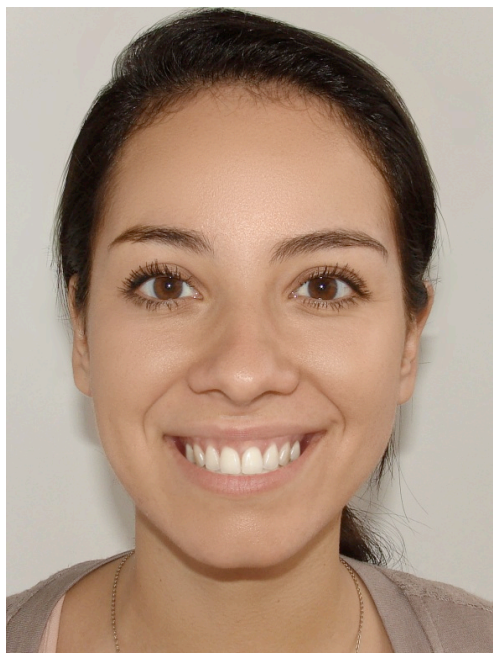

EG 4mm - CB amplio

Figura 1. Modificación de fotografías.

que visualizarían un rotafolio; en el cual primero observarían 9 fotografías por un período máximo de 45 segundos; transcurrido ese tiempo tendrían que elegir una de las fotografías como la más agradable de todas y otra como la menos agradable. Luego se le solicitó al evaluador que comenzara a observar fotografía por fotografía, dispuestas en las siguientes páginas del rotafolio; indicándole que podía evaluarlas por un periodo máximo de 30 segundos cada una; no comparándolas entre sí y valorando cada una de ellas mediante una Escala Visual Análoga (EVA). La encuesta incluyó información como: sexo, edad y profesión del evaluador; constó de dos preguntas con alternativas múltiples y una escala visual análoga (EVA) para calificar el nivel de agrado de cada una de las fotografías. La escala visual análoga (EVA) estuvo compuesta por una línea recta de $10 \mathrm{~cm}$ de longitud que iba de 0 a 10 donde cero es "no me agrada" y 10 "me agrada mucho". Una vez que se recolectaron todas las encuestas, los resultados fueron digitados y codificados en una hoja de cálculo para su posterior análisis.

\section{RESULTADOS}

Los evaluadores fueron 105 personas; de las cuales el 50,5\% $(\mathrm{n}=53)$ perteneció al sexo masculino $y$ el $49,5 \%(n=52)$ al sexo femenino. Asimismo, el promedio de edad fue de $25,73 \pm 4,94$ años; siendo la edad mínima 18 años y la edad máxima 41 años. Cada grupo de evaluadores estuvo conformado por 35 sujetos quienes aceptaron valorar voluntariamente un grupo de fotografías (pertenecientes al rosto de una mujer). El grupo de fotografías incluía 9 fotografías de la misma persona en máxima sonrisa mostrando 
3 diferentes niveles de exposición gingival: $0 \mathrm{~mm}$, $2 \mathrm{~mm}, 4 \mathrm{~mm}$ conjugados con 3 tipos de corredor bucal: amplio, nulo, estrecho.

Para el análisis estadístico de los resultados se utilizó el programa SPSS en su versión de acceso para Windows. En este trabajo de investigación se fijó un nivel de significancia del 5\% con intervalo de confianza del 95\%. En la estadística descriptiva se obtuvieron porcentajes para las variables cualitativas y media con desviación estándar para las variables cuantitativas. Se realizó la prueba de KolnogorovSmirnov para evaluar normalidad; y se encontró un $\mathrm{p}>0,05$ por lo cual se realizaron pruebas paramétricas como Anova Tukey.
Al evaluar la percepción estética sobre los diferentes tipos de corredor bucal y exposición gingival de cada grupo evaluador (personas comunes, Bachilleres y Residentes), medida en una escala cualitativa, se encontró que la sonrisa valorada como la más agradable, en un $9,5 \%$ por las personas comunes fue la que tuvo exposición gingival $0 \mathrm{~mm}$ y corredor bucal estrecho. Mientras que para un 11,4\% por los Bachilleres y un 10,5\% de los Residentes la las agradable fue la que presento exposición gingival 0 $\mathrm{mm}$ y corredor bucal nulo. La sonrisa valorada como la más desagrable; en el 15,2\% de los Bachilleres; el $12,4 \%$ de los Residentes y el $8,6 \%$ de las personas comunes fue la que presentó exposición gingival 4 mm y corredor bucal amplio (Tabla 1).

Tabla 1. Percepción estética de los diferentes tipos de corredor bucal y exposición gingival entre las personas comunes, Bachilleres y Residentes.

\begin{tabular}{|c|c|c|c|c|c|c|}
\hline \multirow[b]{3}{*}{$\begin{array}{l}\text { EG (Exposición gingival) y CB } \\
\text { (Corredor bucal) }\end{array}$} & \multicolumn{6}{|c|}{ Percepción Estética } \\
\hline & \multicolumn{3}{|c|}{ Más agradable } & \multicolumn{3}{|c|}{ Menos agradable } \\
\hline & Persona Común & Bachiller & Residente & Persona Común & Bachiller & Residente \\
\hline EG 2mm - CB amplio & $1,0 \%$ & $2,9 \%$ & $0,0 \%$ & $5,7 \%$ & $1,0 \%$ & $2,9 \%$ \\
\hline EG 4mm - CB nulo & $4,8 \%$ & $1,0 \%$ & $0,0 \%$ & $3,8 \%$ & $7,6 \%$ & $7,6 \%$ \\
\hline EG $2 \mathrm{~mm}-\mathrm{CB}$ nulo & $4,8 \%$ & $2,9 \%$ & $5,7 \%$ & $1,9 \%$ & $0,0 \%$ & $1,0 \%$ \\
\hline EG $0 \mathrm{~mm}-\mathrm{CB}$ estrecho & $9,5 \%$ & $8,6 \%$ & $8,6 \%$ & $1,0 \%$ & $0,0 \%$ & $0,0 \%$ \\
\hline EG $2 \mathrm{~mm}-\mathrm{CB}$ estrecho & $2,9 \%$ & $0,0 \%$ & $3,8 \%$ & $0,0 \%$ & $0,0 \%$ & $0,0 \%$ \\
\hline EG 0mm - CB amplio & $1,9 \%$ & $5,7 \%$ & $4,8 \%$ & $1,9 \%$ & $2,9 \%$ & $3,8 \%$ \\
\hline EG 0mm - CB nulo & $3,8 \%$ & $11,4 \%$ & $10,5 \%$ & $6,7 \%$ & $0,0 \%$ & $0,0 \%$ \\
\hline EG 4mm - CB estrecho & $1,0 \%$ & $1,0 \%$ & $0,0 \%$ & $3,8 \%$ & $6,7 \%$ & $5,7 \%$ \\
\hline EG 4mm - CB amplio & $3,8 \%$ & $0,0 \%$ & $0,0 \%$ & $8,6 \%$ & $15,2 \%$ & $12,4 \%$ \\
\hline
\end{tabular}

Tabla 2. Comparación de la percepción estética sobre los diferentes tipos de corredor bucal y exposición gingival entre las personas comunes, Bachilleres y Residentes.

\begin{tabular}{|c|c|c|c|c|c|c|c|}
\hline \multirow{3}{*}{$\begin{array}{l}\text { EG (Exposición gingival) } \\
\text { y } \\
\text { CB (corredor bucal) }\end{array}$} & \multicolumn{6}{|c|}{ Percepción Estética*(cm) } & \multirow[b]{3}{*}{ Valor $p$} \\
\hline & \multicolumn{2}{|c|}{ Persona Común } & \multicolumn{2}{|c|}{ Bachiller } & \multicolumn{2}{|c|}{ Residente } & \\
\hline & $X^{a}$ & D.S & $\mathrm{X}^{\mathrm{b}}$ & D.S & $X^{c}$ & D.S & \\
\hline EG $2 \mathrm{~mm}$ - CB amplio & 4,32 & 2,58 & 3,95 & 1,62 & 2,93 & 1,87 & $0,017^{\mathrm{a}-\mathrm{b}}$ \\
\hline EG 4mm - CB nulo & 5,05 & 2,49 & 3,03 & 1,81 & 2,86 & 2,34 & $0,000^{\mathrm{a}-\mathrm{bc}}$ \\
\hline EG $0 \mathrm{~mm}-\mathrm{CB}$ estrecho & 5,12 & 2,36 & 5,82 & 1,70 & 6,02 & 1,97 & 0,156 \\
\hline EG $2 \mathrm{~mm}-\mathrm{CB}$ estrecho & 5,07 & 2,51 & 4,87 & 1,37 & 5,21 & 2,08 & 0,786 \\
\hline EG 0mm - CB amplio & 5,40 & 2,33 & 5,69 & 2,49 & 4,68 & 2,67 & 0,230 \\
\hline EG 0mm - CB nulo & 4,85 & 2,52 & 6,85 & 1,69 & 6,78 & 1,76 & $0,000^{\mathrm{a}-\mathrm{bc}}$ \\
\hline EG 4mm - CB amplio & 4,08 & 3,22 & 2,32 & 1,71 & 2,13 & 1,70 & $0,001^{\mathrm{a}-\mathrm{bc}}$ \\
\hline
\end{tabular}

*Valores según escala visual análoga $(0-10 \mathrm{~cm})$; DS: Desviación estándar; Anova, Tukey - p<0,05; <a-b>: La media del grupo a tiene diferencia estadísticamente significativa con la media del grupo b; aplica lo mismo para $<\mathrm{a}-\mathrm{c}>\mathrm{y}<\mathrm{b}-\mathrm{c}>$. $<\mathrm{a}-\mathrm{bc}>$ : la media del grupo a tiene diferencias significativas con las medias del grupo b y c. 
Tabla 3. Comparación de la percepción estética sobre los diferentes tipos de corredor bucal y exposición gingival entre las personas comunes, Bachilleres y Residentes, en el sexo masculino.

\begin{tabular}{|c|c|c|c|c|c|c|c|}
\hline \multirow{3}{*}{$\begin{array}{l}\text { EG (Exposición gingival) y } \\
\text { CB (corredor bucal) }\end{array}$} & \multicolumn{6}{|c|}{ Percepción Estética* $(\mathrm{cm})$ - Sexo Masculino } & \multirow[b]{3}{*}{ Valor $p$} \\
\hline & \multicolumn{2}{|c|}{ Persona Común } & \multicolumn{2}{|c|}{ Bachiller } & \multicolumn{2}{|c|}{ Residente } & \\
\hline & $\mathrm{X}^{\mathrm{a}}$ & D.S & $\mathrm{X}^{\mathrm{b}}$ & D.S & $\mathrm{X}^{\mathrm{c}}$ & D.S & \\
\hline EG $2 \mathrm{~mm}$ - CB amplio & 3,94 & 2,56 & 4,54 & 1,33 & 2,96 & 2,21 & 0,168 \\
\hline EG 4mm - CB nulo & 5,21 & 2,74 & 2,72 & 1,99 & 3,93 & 2,81 & $0,028^{\mathrm{a}-\mathrm{b}}$ \\
\hline EG $2 \mathrm{~mm}-\mathrm{CB}$ nulo & 5,00 & 2,53 & 3,91 & 2,15 & 5,53 & 2,82 & 0,251 \\
\hline EG 0mm - CB estrecho & 4,78 & 2,50 & 6,39 & 1,53 & 6,47 & 2,12 & $0,032^{\mathrm{a}-\mathrm{c}}$ \\
\hline EG $2 \mathrm{~mm}$ - CB estrecho & 4,60 & 2,65 & 5,14 & 1,21 & 5,84 & 2,20 & 0,240 \\
\hline EG 0mm - CB amplio & 5,08 & 2,43 & 5,99 & 1,64 & 4,52 & 2,72 & 0,275 \\
\hline EG 0mm - CB nulo & 4,31 & 2,55 & 6,84 & 1,73 & 6,85 & 1,39 & $0,000^{\mathrm{a}-\mathrm{bc}}$ \\
\hline EG $4 \mathrm{~mm}-\mathrm{CB}$ estrecho & 4,10 & 2,49 & 2,12 & ,95 & 3,49 & 1,65 & $0,025^{\mathrm{a}-\mathrm{b}}$ \\
\hline EG 4mm - CB amplio & 3,58 & 3,20 & 1,99 & 1,01 & 2,18 & 2,00 & 0,110 \\
\hline
\end{tabular}

Tabla 4. Comparación de la percepción estética sobre los diferentes tipos de corredor bucal y exposición gingival entre las personas comunes, Bachilleres y Residentes, en el sexo femenino.

\begin{tabular}{|c|c|c|c|c|c|c|c|}
\hline \multirow{3}{*}{$\begin{array}{l}\text { EG (Exposición gingival) y } \\
\text { CB (Corredor bucal) }\end{array}$} & \multicolumn{6}{|c|}{ Percepción Estética* $(\mathrm{cm})-$ Sexo Femenino } & \multirow[b]{3}{*}{ Valor $p$} \\
\hline & \multicolumn{2}{|c|}{ Persona Común } & \multicolumn{2}{|c|}{ Bachiller } & \multicolumn{2}{|c|}{ Residente } & \\
\hline & $\mathrm{X}$ & D.S & $\mathrm{X}$ & D.S & $\mathrm{X}$ & D.S & \\
\hline EG $2 \mathrm{~mm}-\mathrm{CB}$ amplio & 5,25 & 2,53 & 3,64 & 1,69 & 2,91 & 1,60 & $0,008^{\mathrm{a}-\mathrm{bc}}$ \\
\hline $\mathrm{EG} 4 \mathrm{~mm}-\mathrm{CB}$ nulo & 4,64 & 1,78 & 3,19 & 1,74 & 1,97 & 1,39 & $0,000^{\mathrm{abc}}$ \\
\hline EG $2 \mathrm{~mm}-\mathrm{CB}$ nulo & 5,84 & 2,18 & 4,87 & 1,70 & 4,62 & 2,34 & 0,305 \\
\hline EG $0 \mathrm{~mm}-\mathrm{CB}$ estrecho & 5,96 & 1,79 & 5,52 & 1,73 & 5,64 & 1,81 & 0,809 \\
\hline EG $2 \mathrm{~mm}-\mathrm{CB}$ estrecho & 6,25 & 1,70 & 4,73 & 1,44 & 4,68 & 1,85 & $0,038^{\mathrm{a}-\mathrm{bc}}$ \\
\hline EG 0mm - CB amplio & 6,19 & 1,93 & 5,53 & 2,86 & 4,82 & 2,70 & 0,405 \\
\hline EG 0mm - CB nulo & 6,21 & 1,93 & 6,85 & 1,71 & 6,73 & 2,05 & 0,663 \\
\hline EG 4mm - CB estrecho & 4,92 & 3,08 & 3,29 & 2,01 & 2,68 & 1,38 & $0,027^{\mathrm{a}-\mathrm{c}}$ \\
\hline EG 4mm - CB amplio & 5,33 & 3,06 & 2,49 & 1,98 & 2,08 & 1,46 & $0,001^{\mathrm{a}-\mathrm{bc}}$ \\
\hline
\end{tabular}

Al evaluar la influencia de la percepción estética sobre los diferentes tipos de corredor bucal y exposición gingival entre los 3 grupos de evaluadores; medida con una escala visual análoga $(\mathrm{cm})$, se observó diferencia estadísticamente significativa en las percepciones entre los tres grupos de evaluadores (Anova- Tukey; $\mathrm{p}<0,05$ ) (Tablas 2,3 y 4).

\section{DISCUSIÓN}

La influencia tanto del corredor bucal y la sonrisa gingival han sido investigadas en numerosos estudios con anterioridad (15-25). Sin embargo, estos generalmente se centraron en una sola característica de la sonrisa estética no investigando la interacción entre ellas. Es así, que mediante el presente estudio se buscó obtener información sobre la influencia que ejercen el corredor bucal y la exposición gingival sobre la percepción de la sonrisa en nuestra población, mediante la utilización de encuestas.

Se utilizó la escala visual análoga (EVA) para juzgar el atractivo de la sonrisa; ya que con anterioridad su uso en la puntuación de la estética, proporcionando resultados simples, rápidos y reproducibles $(15,17,18,20,21,25,27,28)$. Además se usó el método de modificación de fotografías mediante el programa Adobe Photoshop CS6 como en estudios similares $(15,16,18,20,22,23,25-27,29,30)$.

Estudios anteriores $(15,21,22,23)$ que evaluaron la influencia de la exposición gingival en la percepción 
de la sonrisa, utilizaron modificaciones de $-5 \mathrm{a}+5$ $\mathrm{mm}$ con incrementos de $1 \mathrm{~mm}$ (21), de 0 a $7 \mathrm{~mm}$ con incrementos de $2 \mathrm{~mm}$ (23), de -2 a $+4 \mathrm{~mm}$ con incrementos de $1 \mathrm{~mm}$ (15) e incrementos de $2 \mathrm{~mm}$ (22). Sin embargo en este estudio se comparó la exposición gingival mediante la modificación solo de tres niveles diferentes, los cuales fueron: $0 \mathrm{~mm}$, $+2 \mathrm{~mm}$ y $4 \mathrm{~mm}$ de cobertura del labio superior a los incisivos centrales superiores, debido a que se decidió elegir las medidas mayormente utilizadas en estos estudios; siempre que no fueran tan extremas y que sean posibles de corregir durante el tratamiento ortodóncico sin necesidad de tratamiento quirúrgico. Además, por la literatura se conoce que exposiciones mayores a $4 \mathrm{~mm}$ son consideradas estéticamente desagradables, por lo que no fueron considerados rangos mayores.

Debido a que muchos factores podían ser factor de confusión para los sujetos evaluadores, se conjugo los tres tipos de exposición gingival elegidos con otros tres tipos de corredor bucal que fueron: corredor bucal estrecho, corredor bucal amplio y corredor bucal nulo (sin presencia de corredor bucal). Al igual que en el estudio de Parekh y col. (17) y diferente a estudios de Moore y col. (16), Zange y col. (24) y Loi y col. (25) donde clasificaron al corredor bucal en porcentajes.

Existen algunos estudios en los que se evaluó de forma independiente la influencia del corredor bucal o la exposición gingival sobre la población peruana; como es el de Flores y col. (28), pero no existen datos suficientes para determinar lo que es estéticamente atractivo en la percepción de la sonrisa, por lo que este estudio sería uno de los primeros que hace una combinación de dos variables sobre el atractivo de la sonrisa, cuando es juzgada por Residentes de Ortodoncia, Bachilleres en Estomatología y personas comunes que pueden acudir a la consulta dental, ya que uno de nuestros objetivos fue determinar si el nivel educativo o el grado de instrucción puede alterar la percepción de la sonrisa.

Los resultados se basaron en las respuestas de 105 sujetos de estudio; 35 fueron personas comunes, 35 Bachilleres en Estomatología y 35 Residentes de Ortodoncia; formando tres grupos comparables entre sí, con un número de evaluadores similares a los estudios de Brum y col. (22) que evaluaron 90 sujetos, siendo los evaluadores: 30 personas comunes, 30 odontólogos y 30 ortodoncistas, y Kaya y col. (27) que evaluaron un total de 168 sujetos (56 por grupo) ortodoncistas, odontólogos y personas comunes. A diferencia de los estudios de Hunt y col. (15) que sólo evaluaron 120 personas comunes y Susuki y col. (23) que evaluaron 60 personas (ortodoncistas, cirujanos buco-maxilo-faciales y personas comunes).

Este estudio demostró que al evaluar la agradabilidad de la sonrisa personas comunes, Bachilleres y Residentes (Tabla1), la mayoría las personas comunes eligieron como la sonrisa más agradable la que presentó una EG $0 \mathrm{~mm}$ - CB estrecho con un 9,5\% de los casos; mientras que Bachilleres y Residentes consideraron como la sonrisa más agradable la que presentó EG $0 \mathrm{~mm}$ - CB nulo; en $11,4 \%$ y $10,5 \%$ de los casos. Estudios similares dan tambien las mayores valoraciones a la EG de 0 $\mathrm{mm}$; así Hunt y col. (15) encontraron que para las personas comunes la sonrisa con EG 0mm fue calificada como la más atractiva, Brum y col. (22) encontraron que ortodoncistas, odontólogos y personas comunes prefirieron sonrisas con EG 0m y Susuki y col. (23) encontraron que una EG de $0 \mathrm{~mm}$ y $1 \mathrm{~mm}$ fueron elegidas como las más atractivas por ortodoncistas, cirujanos buco-maxilo-faciales y personas comunes. Por otro lado, al evaluar corredor bucal, el estudio de Moore y col. (15) encontraron que las personas comunes prefirieron que durante la sonrisa se exponga un mínimo de corredor bucal (CB 2\%) al igual que en el presente estudio donde las personas comunes prefirieron corredores bucales estrechos. Asimismo, Martin y col. (7) encontraron que ortodoncistas prefirieron corredores nulos como los más atractivos, mientras que las personas comunes prefieren corredores bucales estrechos.

Por otro lado, en el presente estudio la mayoría de personas comunes, Bachilleres y Residentes de Ortodoncia, eligieron como sonrisa menos agradable la que presentó EG $+4 \mathrm{~mm}-\mathrm{CB}$ amplio con $8,6 \%$, $15,2 \%$ y $12,4 \%$ respectivamente. Comparable para el caso de la exposición gingival con el estudio de Brum y col. (12) donde la sonrisa menos agradable elegida por ortodoncistas, odontólogos y personas comunes, fue la que tuvo una $\mathrm{EG}+4 \mathrm{~mm}$. Hunt y col. (15) encontraron como menos agradable la $\mathrm{EG}+3 \mathrm{~mm}$ y $+4 \mathrm{~mm}$ y Susuki y col.(23) sonrisas con $\mathrm{EG}$ de +3 , $+5 \mathrm{y}+7 \mathrm{~mm}$. Y para el caso del corredor bucal los resultados del presente estudio son similares a los estudios de Moore y col. (3), Martin y col. (7) y Ritter 
y col. (18).

Al evaluar la agradabilidad de la sonrisa de personas comunes, Bachilleres y Residentes según el sexo, se encontró que tanto para el sexo femenino como masculino la sonrisa elegida como la más agradable por las personas comunes fue la que presento EG $0 \mathrm{~mm}$ - CB estrecho con un 9,4\% y $9,6 \%$, mientras que Bachilleres y Residentes eligieron como más agradable EG $0 \mathrm{~mm}$ - CB nulo con 7,5\% y $9,4 \%$ para varones $-15,4 \%$ y $11,5 \%$ para mujeres respectivamente. La sonrisa elegida como la menos agradable para el sexo masculino en opinión de personas comunes, Bachilleres y Residentes fueron las sonrisas con EG 4mm - CB amplio. Para el sexo femenino la sonrisa menos agradable fue la que presento EG 4mm - CB amplio además de CB nulo y estrecho en el caso de Residentes.

Estos últimos datos coinciden con los de la Tabla1, demostrando que no hay diferencia en la elección de la sonrisa más agradable en cuanto al sexo del evaluador. Siendo la exposición gingival de $0 \mathrm{~mm}$ la más agradable para todos los grupos, y la de $4 \mathrm{~mm}$ la menos agradable; difiriendo en el agrado del corredor bucal donde personas comunes prefieren como más atractivo $\mathrm{CB}$ estrechos y Bachilleres y Residentes CB nulos, ninguno de los grupos prefieren $\mathrm{CB}$ amplios. Esto es similar que en el estudio de Moore y col. (16) donde personas comunes prefieren corredores bucales mínimos como más agradables, no encontrando diferencia significativa entre el juicio de hombre y mujeres. Siendo los corredores amplios considerados no agradables; y contrario al estudio de Elham y col. (30) quienes observaron que los evaluadores masculinos y femeninos puntuaron a las fotografías con exposición gingival de manera diferente, sugiriendo que los evaluadores femeninos son más tolerantes a la exposición gingival.

Zange y col. (4) sostienen que la presencia o ausencia de corredores tiene poca influencia sobre la estética de la sonrisa, no habiendo justificación para eliminar oscuros espacios a menos que sean muy evidentes. Al igual Roden y col. (26) afirman que la presencia de corredores bucales no tiene influencia en la estética de la sonrisa.

La mayoría de estudios hacen la comparación de la percepción en cuanto al sexo de un solo grupo evaluador mas no comparan en función a diferentes grupos de evaluadores como en el presente estudio donde se quiso ver si de alguna manera el nivel educativo acerca de connotaciones estéticas alteró la percepción de la sonrisa, por lo que al determinar la influencia de los tipos de corredor bucal y exposición gingival sobre la percepción estética de la sonrisa evaluada mediante una Escala Visual Análoga entre personas comunes, Bachilleres y Residentes (Tabla3), se encontró una diferencia estadísticamente significativa entre los grupos (Anova - Tukey; $\mathrm{p}<0,05$ ), donde mayormente las personas comunes difirieron de los Bachilleres o de los Residentes en cuanto a sus criterios estéticos de percepción de la sonrisa, mientras que Bachilleres y Residentes si coinciden respecto a su percepción; lo cual puede ser debido a que Bachilleres y Residentes al llevar cursos tienen criterios de estética más perceptibles y similares.

A la hora de verificar las puntuaciones dadas por los grupos evaluadores a los 9 tipos de sonrisas creadas, las personas comunes tuvieron percepciones muy dispersas ya que por un lado le dieron mayor puntuación a la EG de $0 \mathrm{~mm}$ y $\mathrm{CB}$ amplio y por otro a la EG $2 \mathrm{~mm}$ y $\mathrm{CB}$ nulo lo que denota que tienen muy dispersa su apreciación estética con una media de 5,40 y 5,24 esto pudo haber sido a que requerían observar características muy marcadas.

Los Bachilleres y Residentes en su mayoría se inclinaron a dar una mayor puntuación por una $\mathrm{EG}$ $0 \mathrm{~mm}$ y un $\mathrm{CB}$ nulo con medias de 6,85 y 6,78 en relación a las personas comunes las cuales tuvieron una media de 4,85 donde se observó que la media del grupo de personas comunes tuvo diferencias estadísticamente significativas con las medias de los grupos de Bachilleres y Residentes. Sin embargo, en su mayoría las sonrisas con mayor puntuación fueron dadas por las personas comunes en comparación con Bachilleres y Residentes los cuales frente a sonrisas que no están dentro de sus parámetros; atribuyen puntuaciones menores y similares. Estos resultados fueron similares al estudio de Martin y col. (19) donde las personas comunes fueron menos exigentes que los ortodoncistas en sus percepciones. Las personas comunes valoraron con mayor puntaje que Bachilleres y Residentes las sonrisas con exposiciones gingivales de $2 \mathrm{~mm}$ y $4 \mathrm{~mm}$; aceptando los tres tipos de corredores bucales, lo que significa que personas comunes son 
más tolerantes. Mientras que Bachilleres y Residentes valoraron con puntuaciones altas solo EG $0 \mathrm{~mm}$ y CB nulo de acuerdo a las normas establecidas. Esto difiere de Zange y col. (24) donde las personas comunes son más críticas en su evaluación que los ortodoncistas. Al evaluar la influencia del sexo de los diferentes grupos de sujetos encuestados sobre la percepción estética (Tablas 3 y 4) también se encontró diferencias estadísticamente significativas tanto para el sexo masculino como femenino entre los grupos (Anova Tukey; $\mathrm{p}<0.05$ ); donde para la variedad de sonrisas; mayormente las personas comunes del sexo masculino y femenino tienen percepciones distintas a las de Bachilleres, de Residentes, a ambos grupos, e incluso hay diferencias significativas entre los tres grupos.

Se observó que en el sexo femenino las personas comunes dieron las mayores puntuaciones a la EG $2 \mathrm{~mm} \mathrm{CB}$ estrecho con una media de 6,25 a diferencia de Bachilleres que dieron una media de 4,73 y Residentes 4,68. Corroborando las diferencias de percepción entre el grupo de personas comunes con Bachilleres y Residentes que poseen en este caso percepciones similares para este tipo de sonrisa; dando puntuaciones menores. Igual sucedió para el sexo masculino donde personas comunes dieron su mayor puntuación a la EG $4 \mathrm{~mm} \mathrm{CB}$ nulo con una media de 5,21 contrario a Bachilleres con 2,72 y Residentes con 3,93.

Como un hallazgo del estudio, al evaluar las puntuaciones más altas dadas por las personas comunes, se encontró que: el sexo masculino (media de 5,21 para la EG 4mm CB nulo) es más tolerante a exposiciones gingivales de $4 \mathrm{~mm}$ y corredores bucales nulos a diferencia del sexo femenino que toleran mejor las exposiciones gingivales de $2 \mathrm{~m}$ y corredores bucales mínimos o estrechos (media de 6,25); dando menores puntuaciones ante exposiciones gingivales de $4 \mathrm{~mm}$ y corredores bucales nulos (media de 4,64). De lo cual se podría concluir que hay diferencia entre personas comunes del sexo masculino y femenino, lo cual puede ser que al utilizar la fotografía de una mujer, el sexo opuesto, haya puntuado de manera variada su elección. Algunos con la percepción enfocada hacia una sonrisa femenina y otros hacia una sonrisa masculina.

Elham y col. (30) y Geron y col. (31) observaron que los evaluadores masculinos y femeninos puntuaron las fotografías con exposición gingival de manera diferente, sugiriendo que los evaluadores femeninos son más tolerantes a la exposición gingival. Contrario con los resultados de nuestro estudio, pues los evaluadores masculinos dieron sus mayores puntuaciones a la exposición gingival de $4 \mathrm{~mm}$ y las femeninas a la exposición gingival de $2 \mathrm{~mm}$.

Es importante destacar que los ortodoncistas siempre se esforzarán por lograr el estándar estético ideal en sus pacientes, siempre y cuando los resultados obtenidos los satisfagan cumpliendo sus expectativas estéticas a pesar de las dificultades y limitaciones que existen en cualquier tratamiento odontológico.

Las limitaciones de este estudio incluyeron el uso de una sonrisa femenina como único modelo de la imagen, pues se ha demostrado que el género de la imagen afecta la puntuación en el atractivo de una sonrisa (31). Además, aunque la muestra fue comparativa y similar a otros estudios, se aconseja procurar homogenizar la cantidad de participantes tanto por género, edad y grado de instrucción para obtener valores más proporcionales y confiables.

Otras de las limitaciones, fue que no se tuvo en la cuenta la condición socioeconómica de los encuestados. Puede ser que esta variable haya afectado los resultados de una manera importante.

Por lo tanto se necesitan más estudios, con muestras más numerosas y con la introducción de nuevas variables para explorar el tema, poco investigado en nuestro país pero de gran interés debido a la gran diversidad racial y cultural observada en el Perú.

Se puede concluir que, personas comunes, Bachilleres y Residentes prefieren sonrisas con exposiciones gingivales mínimas a nulas y corredores bucales mínimos a nulos. Considerando sonrisas con corredores bucales amplios y exposiciones gingivales de $4 \mathrm{~mm}$ como sonrisas no atractivas. Tanto el sexo masculino como el sexo femenino coinciden en considerar desagradables las exposiciones gingivales de $+4 \mathrm{~mm}$, pero el agrado o desagrado del corredor bucal según el sexo está relacionado con la cantidad exposición gingival que se muestre, apreciándose que el corredor bucal no tiene una influencia relevante sobre la percepción de la sonrisa. Se encontraron 
diferencias estadísticamente significativas al juzgar la atracción de la sonrisa entre los grupos, donde las personas comunes difieren de la percepción de los Bachilleres y Residentes, ya que estos están relacionados a su formación.

\section{Correspondencia:}

\section{Katherine Rose Espinoza Barco}

Dirección: Av. Próceres de la Independencia 3361-

San Juan de Lurigancho

Email: k.rose_16@outlook.com

\section{REFERENCIAS BIBLIOGRÁFICAS}

1 Dong JK, Jin TH, Cho HW, Oh SC. The esthetics of smile: a review of some recent studies. The International Journal of Prosthodontics 1999; 12(1):9-19.

2 Padrós E. Bases diagnósticas, terapéuticas y posturales del funcionalismo craneofacial. Madris: Ed. Ripano; 2006. P. 450

3 Baumgarten AG. Meditationes pholosophicae de nonnullis and poema pertinentibus, 1735. In: Aschenbrenner k, Holther WB, translators. Reflections on poetry. Berkeley: University of California Press, 1954.

4 Proffit \& White. The search for truth: diagnosis. En: Surgical Orthodontic Treatment. Ed.Mosby. 1991; 93141.

5 Proffit. Diagnóstico y planificación del tratamiento. En: Ortodoncia Teoría y Práctica. Ed. Mosby. 1994; 143-9.

6 Pinho S, Ciriaco C, Faber J, Lenza MA. Impact of dental asymmetries on the perception of smile esthetics. Am J Orthod Dentofacial Orthop. 2007; 132(6):74853.

7 Carbone D. Análisis de los ángulos de convexidad facial y nasolabial en fotografías de niños respecto a sus distintos estadios de dentición en oclusión normal y maloclusiones según Angle de la clínica Estomatológica central de la Universidad Peruana Cayetano Heredia entre los años 2006-2010. [Tesis para optar el Título de Cirujano Dentista]. Lima: Universidad Peruana Cayetano Heredia. Facultad de Estomatología; 2011.

8 Dustin R J, Gallerano R. English J. The effects of bucal corridor spaces and arch form on smile esthetics. Am J Orthod Dentofacial Orthop. 2003; 127 (3):343-350.

9 Heravi F, Rashed R, Abachizadeh H. Esthetic preferences for the shape of anterior teeth in a posed smile. Am J Orthod Dentofacial Orthop. 2011; 139(6):806-14.

10 Shaw WC. Factors influencing the desire for orthodontic treatment. Eur J Orthod. 1981; 3(3):151-62.

11 Espeland LV, Stenvik A. Perception of personal dental appearance in young adults: relationship between occlusion, awareness, and satisfaction. Am J Orthod Dentofacial Orthop. 1991; 100(3):234-41.

12 Sarver DM. The importance of incisor position in the esthetic smile: the smile arc. Am J Orthod Dentofacial Orthop. 2001; 120(2):98-111.

13 Ackerman JL, Proffit WR, Sarver DM. The emerging softtissueparadigminorthodonticdiagnosisandtreatment planning. Clin Orthod Res. 1999; 2(2):49-52.

14 Ioi H, Nakata S, Counts AL. Effects of buccal corridors on smile esthetics in Japanese. Angle Orthod. 2009; 79(4):628-33.

15 Hunt O, Johnston C, Hepper P, Burden D, Stevenson M. The influence of maxillary gingival exposure on dental attractiveness ratings. Eur J Orthod. 2002; 24(2):199-204.

16 Moore T, Southard KA, Casko JS, Qian F, Southard TE. Buccal corridors and smile esthetics. Am J Orthod Dentofacial Orthop. 2005; 127(2):208-13.

17 Parekh SM, Fields HW, Beck M, Rosenstiel S. Attractiveness of variations in the smile arc and buccal corridor space as judged by orthodontists and laymen. Angle Orthod. 2006; 76(4):557-63.

18 Ritter DE, Gandini LG, Pinto Ados S, Locks A. Esthetic influence of negative space in the buccal corridor during smiling. Angle Orthod. 2006; 76(2):198-203.

19 Martin AJ, Buschang PH, Boley JC, Taylor RW, McKinney TW. The impact of buccal corridors on smile attractiveness. Eur J Orthod. 2007; 29(5):530-7.

20 Suzuki L, Wilson A, Machado, Vieira M. Perceptions of gingival display aesthetics among orthodontists, maxillofacial surgeons and laypersons. Rev Odonto Ciênc. 2009; 24(4):367-71.

21 Loi H, Nakata S, Counts AL. Influence of gingival display on smile aesthetics in Japanese. Eur $\mathrm{J}$ Orthod. 2010; 32(6):633-7.

22 Brum M, Enéas D, Borgatto A, D'Agostine C, Rocha R. Influência da exposição gengival na estética do sorriso. Dental Press J Orthod. 2011; 16(5):111-8.

23 Suzuki L, Machado A, Vieira M. Avaliação da influência da quantidade de exposição gengival na estética do sorriso. Dental Press J Orthod. 2011; 16(5): 37.e 1-10.

24 Zange SE, Ramos AL, Cuoghi OA, de Mendonça MR, Suguino R. Perceptions of laypersons and orthodontists regarding the buccal corridor in long- and short-face individuals. Angle Orthod. 2011; 81(1):8690

25 Loi H, Kang S, Shimomura T, Kim S, Park S, Son W, Takahashif I. Effects of buccal corridors on smile esthetics in Japanese and Korean orthodontists and orthodontic patients. Am J Orthod Orthop dentofacial. 2012; 142:459-65.

26 Roden-Johnson D, Gallerano R, English J. The effects of buccal corridor spaces and arch form on smile esthetics. Am J Orthod Dentofacial Orthop. 2005; 
127(3):343-50.

27 Kaya B, Uyar R. Influence on smile attractiveness of the smile arc in conjunction with gingival display. Am J Orthod Dentofacial Orthop. 2013; 144(4):541-7.

28 Flores Vignolo R, Meneses López A, Liñán Durán C. Influencia de la exposición gingival en la percepción estética de la sonrisa. Rev Estomatol Herediana. 2013; 23(2):76-82.

29 Rodrigues C de D, Magnani R, Machado MS, Oliveira OB. The perception of smile attractiveness. Angle Orthod. 2009; 79(4):634-9.
30 Elham S. J Abu Alhaija, Nada O. Al-Shamsi, Susan Al-Khateeb. Perceptions of Jordanian laypersons and dental professionals to altered smile aesthetics. Eur J Orthod. 2011; 33(4):450-6.

31 Geron S, Atalia W. Influence of sex on the perception of oral and smile esthetics with differente gingival display and incisal plane inclination. Angle Orthod. 2005; 75:778-84

Recibido: 12/01/2015

Aceptado: 05/05/2015 